Conclusion: This study suggests that RA patients from Mexico have high prevalence of the FM. Those with FM have a worse functional status, a higher frequency and score of comorbidities that impact in a reduction of their quality of life. On the other hand, no differences were found for RA disease activity in both groups. However, these observations must be confirmed in larger and prospective studies. REFERENCES:

[1] Zhao SS et al. Best Pract Res Clin Rheumatol. 2019;33(3):101423;

[2] Duffield SJ et al. Rheumatology (Oxford). 2018;57(8):1453;

[3] Kim H et al. Arthritis Care Res (Hoboken) 2017;69(12):1871;

[4] Salaffi Fet al. Rheumatol Int. 2017;37(12):2035.

Disclosure of Interests: None declared

DOI: 10.1136/annrheumdis-2021-eular.1013

\section{POS0516 REDEFINING THE CLINICAL AND LABORATORY FEATURES OF RHEUMATIC PLEURAL EFFUSION: A 30-CASE SERIES}

S. Minoda ${ }^{1}$, R. Sada ${ }^{2}$, S. Matsushita ${ }^{2}$, Y. Nakayama ${ }^{3}$, H. Akebo ${ }^{2}$, Y. Tsugihashi ${ }^{4}$, H. Ishimaru ${ }^{2}, \mathrm{~K}$. Hatta ${ }^{2} .{ }^{1}$ Osaka University Graduate School of Medicine, Department of Respiratory Medicine and Clinical Immunology, Osaka, Japan; ${ }^{2}$ Tenri Hospital, Department of General Internal Medicine, Nara, Japan; ${ }^{3}$ Graduate School of Medicine, Kyoto University, Department of Rheumatology and Clinical Immunology, Kyoto, Japan; ${ }^{4}$ Tenri Health Care University, Department of Health Care, Nara, Japan

Background: Rheumatoid pleural effusion (RPE) is a common extra-articular complication in patients with rheumatoid arthritis (RA). Previous studies have shown that RPE usually occurs in middle-aged men with rheumatoid factor (RF)-positive RA. RPE usually has features of pleural fluid acidosis, high lactate dehydrogenase (LDH) levels, and very low glucose levels ${ }^{(1)}$. However, to the best of our knowledge, these findings were based on very few case series and reports, and most of these reports were published by the early $2000 \mathrm{~s}^{(1,2)}$.

Objectives: To investigate the clinical and laboratory characteristics and typical clinical courses of patients with RPE in a single centre of Japan since the beginning of the 21st century.

Methods: Medical records of RPE patients were retrospectively reviewed between May 2006 and September 2020. RPE was identified by fulfilling these five conditions: (1) confirmation of the RA diagnosis; (2) having an exudative pleural effusion according to Light's criteria; (3) negative results of pleural fluid culture; (4) negative results of pleural fluid cytology; and (5) exclusion of a parapneumonic effusion or empyema defined as no antibiotic use or ineffectiveness of antibiotics during the clinical course. Patients were divided into two groups according to their age at diagnosis: $<60$ years (Group A) and $\geq 60$ years (Group B). Results: A total of 30 cases of RPE were included in the study. The median age was 71 years (interquartile range [IQR], 66-78 years). Of these patients, $16(53 \%)$ were women. The median disease duration of RA was 98 months (IQR, 8-162 months). The two groups comprised six patients aged $<60$ years old and 24 patients $\geq 60$ years. The median age was 54 years (IQR, 49-56 years) in Group A and 74 years (IQR, 69-78 years) in Group B. The median disease duration of RA was longer in Group B than that in Group A (132 vs. 3 months, $p=0.008$ ). Compared with Group A, Group B had fewer patients with fever ( $14 \%$ vs. $83 \%, p=0.003)$, and had lower serum C-reactive protein levels (3.3 vs. $11.1 \mathrm{mg} / \mathrm{dL}, \mathrm{p}=0.03$ ). Moreover, Group B was more likely to show mild inflammatory pleural fluids with higher $\mathrm{pH}(7.5$ vs. $7.2, \mathrm{p}=0.005)$ and lower $\mathrm{LDH}$ levels (155 vs. $1810 \mathrm{IU} / \mathrm{L}, \mathrm{p}=0.046)$. Corticosteroids were started or increased in five (83\%) and nine $(38 \%)$ patients, and biologic disease-modifying anti-rheumatic drugs were started in one (17\%) and two (8\%) patients in groups A and B, respectively. One patient (16\%) died within 5-years in Group A, and seven patients (29\%) died in Group B. Conclusion: In contrast to previous studies, RPE was seen in older patients as well as middle-aged adults, and the pleural fluid analysis in older patients with RPE showed milder inflammation than the middle-aged patients.

Table 1. Comparison of clinical and laboratory findings between Group A and Group B.

\begin{tabular}{|c|c|c|c|}
\hline & Group A $(n=6)$ & Group B $(n=24)$ & $\begin{array}{c}P \\
\text { value }\end{array}$ \\
\hline$\overline{\text { Age (years) }}$ & 54 [49-56] & 74 [69-78] & \\
\hline Female & $2(n=6,33.3)$ & $14(n=24,58.3)$ & 0.38 \\
\hline Disease duration of RA (months) & $3[1-9]$ & $132[44-199]$ & 0.008 \\
\hline Fever $\geq 37.0^{\circ} \mathrm{C}$ & $5(n=6,83.3)$ & $3(n=22,13.6)$ & 0.003 \\
\hline $\begin{array}{l}\text { Serum } \\
\text { CRP (mg/dL) }\end{array}$ & $11.1[5.6-1.4]$ & $3.3[0.9-10.5]$ & 0.03 \\
\hline $\mathrm{RF}(\mathrm{IU} / \mathrm{mL})$ & $100[19-816]$ & $63[23-193]$ & 0.95 \\
\hline $\begin{array}{l}\text { Anti-CCP ab positive } \\
\text { Pleural fluid analysis }\end{array}$ & $5(n=6,83.3)$ & $12(n=15,80)$ & 1.00 \\
\hline $\mathrm{pH}$ & $7.2[7.2-7.2]$ & $7.5[7.4-7.5]$ & 0.005 \\
\hline $\mathrm{LDH}(\mathrm{IU} / \mathrm{L})$ & $1810[594-2932]$ & $155[123-346]$ & 0.046 \\
\hline $\mathrm{Glu}(\mathrm{mg} / \mathrm{dL})$ & $59[10-123]$ & $105[91-122]$ & 0.42 \\
\hline$T p(g / d L)$ & $5.1[4.9-5.6]$ & $4.6[3.6-5.2]$ & 0.21 \\
\hline Number of cells $(/ \mu \mathrm{L})$ & $5235[3353-9300]$ & $3300[1490-5008]$ & 0.27 \\
\hline Glu/serum Glu & $0.41[0.09-0.99]$ & $1.05[0.85-1.15]$ & 0.71 \\
\hline Started or increased CS & $5(n=6,83.3)$ & $9(n=24,37.5)$ & 0.18 \\
\hline Started bDMARDs & $1(n=6,16.6)$ & $2(n=24,8.3)$ & 0.50 \\
\hline Died within 5 years & $1(n=6,16.6)$ & $7(n=24,29.1)$ & 1.00 \\
\hline
\end{tabular}

Data are median [interquartile range], or number (total number, percent).Abbreviations: RA, rheumatoid arthritis; CRP, C-reactive protein; RF, rheumatoid factor; Anti-CCP ab, anti-cyclic citrullinated peptide antibodies; LDH, lactate dehydrogenase; Glu, glucose; Tp, total protein; $\mathrm{CS}$, corticosteroid; bDMARDs, biologic disease-modifying anti-rheumatic drugs
REFERENCES:

[1] Balbir-Gurman A, et al. Semin Arthritis Rheum. 2006 Jun; 35(6): 368-78. Figure. Comparison of pleural fluid lactate dehydrogenase (LDH) and $\mathrm{pH}$ between Group A and Group B.
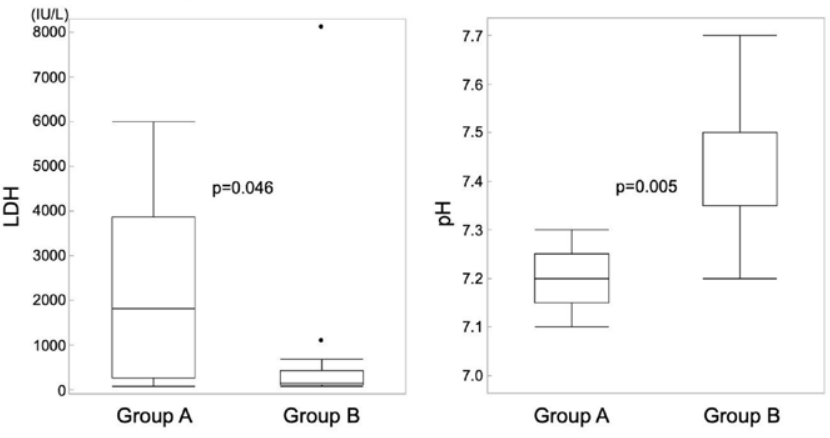

Disclosure of Interests: None declared DOI: 10.1136/annrheumdis-2021-eular.1197

\section{\begin{tabular}{|l|l}
\hline POS0517 & A LONGITUDINAL STUDY OF SARCOPENIA,
\end{tabular} LOCOMOTIVE SYNDROME, AND FRAILTY IN PATIENTS WITH RHEUMATOID ARTHRITIS: FROM THE CHIKARA STUDY}

K. Mandai ${ }^{1}$, M. Tada ${ }^{2}$, Y. Yamada ${ }^{3}$, T. Koike ${ }^{4,5}$, T. Okano $^{3}$, N. Hidaka ${ }^{2}$,

H. Nakamura ${ }^{3}$. ${ }^{1}$ Osaka Saiseikai Nakatsu Hospital, Department of Orthopedic Surgery, Osaka, Japan; ${ }^{2}$ Osaka City General Hospital, Department of Orthopedic Surgery, Osaka, Japan; ${ }^{3}$ Osaka City University Graduate School of Medicine, Department of Orthopedic Surgery, Osaka, Japan; ${ }^{4}$ Osaka City University Graduate School of Medicine, Center for Senile Degenerative Disorders (CSDD), Osaka, Japan; ${ }^{5}$ Search Institute for Bone and Arthritis Disease (SINBAD), Department of Orthopedic Surgery, Shirahama, Japan

Background: Rheumatoid arthritis (RA) patients have a high frequency of sarcopenia and they commonly have reduced physical function. We previously reported that the prevalence of sarcopenia was $28 \%$, that of frailty was $18.9 \%$, and that of pre-frailty was $38.9 \%$ in RA patients ${ }^{1,2}$, and $13.2 \%$ of RA patients developed sarcopenia within a year ${ }^{3}$. Objectives: To investigate the risk factors for new onset of sarcopenia, locomotive syndrome, and frailty in patients with RA and the course of each disease. Methods: Two-year follow-up data from the rural group of the prospective, observational CHIKARA study were used. Sarcopenia was diagnosed using the criteria of the Asian Working Group for Sarcopenia 2014, locomotive syndrome was diagnosed using locomotive 5 , and frailty was diagnosed using the basic checklist. New onset of the disease over the 2-year follow-up period was studied, excluding cases that had the disease at baseline. Improvement was defined as cases with disease at baseline that no longer met the diagnostic criteria after 2 years. Differences in the characteristics of each disease were tested using the Chi-squared test and the paired $t$-test. Results: The 81 patients with RA ( $82.7 \%$ female) had mean age $66.9 \pm 11.5$ years, mean DAS28-ESR $2.9 \pm 1.2$, methotrexate use in $81.5 \%$ (with a dose of $9.9 \pm 2.7 \mathrm{mg}$ / week), and glucocorticoid (GC) use in $22.2 \%$ (with a dose of $3.1 \pm 1.7 \mathrm{mg} /$ week). The baseline prevalence was $44.4 \%$ for sarcopenia, $35.8 \%$ for locomotive syndrome, and $25.9 \%$ for frailty, and the new onset rate was $4.4 \%$ for sarcopenia, $15.4 \%$ for locomotive syndrome, and $13.3 \%$ for frailty. Of the patients with each disease at baseline, $36.1 \%$ had sarcopenia, $20.7 \%$ had locomotive syndrome, and $33.3 \%$ had frailty, and of those with each disease at 2 years, $36.1 \%$ had sarcopenia, $20.7 \%$ had locomotive syndrome, and $33.3 \%$ had frailty. The new onset sarcopenia and locomotive syndrome groups had significantly higher rates of GC use $(p=0.036$, $\mathrm{p}=0.007$, paired $t$-test) and significantly higher doses $(\mathrm{p}=0.01, \mathrm{p}=0.001$, paired $t$-test $)$ than the groups without new onset sarcopenia and locomotive syndrome. High baseline disease activity was an independent predictor of new onset of locomotive syndrome on multivariate logistic regression analysis $(O R=3.21, p=0.015)$.

Conclusion: The new onset rates at 2 years were $4.4 \%$ for sarcopenia, $15.4 \%$ for locomotive syndrome, and $13.3 \%$ for frailty. In the new onset sarcopenia and locomotive syndrome groups, both GC use and dosage were significantly higher. REFERENCES:

[1] Tada $M$, et al. Matrix metalloprotease 3 is associated with sarcopenia in rheumatoid arthritis - results from the CHIKARA study. Int $\mathrm{J}$ Rheum Dis. 2018 Nov;21(11):1962-1969.

[2] Tada $M$, et al. Correlation between frailty and disease activity in patients with rheumatoid arthritis: Data from the CHIKARA study. Geriatr Gerontol Int. 2019 Dec;19(12):1220-1225.

[3] Yamada Y, et al. Glucocorticoid use is an independent risk factor for developing sarcopenia in patients with rheumatoid arthritis: from the CHIKARA study. Clin Rheumatol. 2020 Jun;39(6):1757-1764.

Disclosure of Interests: None declared

DOI: 10.1136/annrheumdis-2021-eular.1245 